\title{
Concurso de Investigación SOCHIPE 2008
}

\author{
PAUL R. HARRIS D. ${ }^{1}$ y FRANCISCO MORAGA M. ${ }^{2}$ \\ 1. Presidente Comisión Investigación, Sociedad Chilena de Pediatría; Comité Editorial Revista Chilena de Pediatría. \\ 2. Directorio Sociedad Chilena de Pediatría.
}

La Sociedad Chilena de Pediatría (SOCHIPE), cumpliendo sus objetivos fundacionales, ha dado un nuevo impulso al desarrollo de la investigación en pediatría, reformulando la estructura del Concurso de Financiamiento de Proyectos de Investigación iniciado el año 2006. Para ello, el año 2008 se llamó a un concurso para financiar trabajos de investigación por un plazo máximo de 2 años, con la finalidad de estimular y apoyar las propuestas de los socios para la ejecución de investigaciones clínicas y epidemiológicas, relacionadas con la salud infantil y del adolescente, que en otros concursos habitualmente quedan postergadas a menos que usen tecnologías de avanzada que aseguren publicaciones con índice de impacto tipo ISI.

El objetivo de este nuevo concurso recién finalizado, fue estimular la actividad de investigación entre los miembros de SOCHIPE, entendiendo como tal el desarrollo de una idea original en un proyecto que sea ejecutable en el plazo de dos años. Sólo se financiaron proyectos de investigación originales, que conduzcan a la producción de nuevos conocimientos, mediante la aplicación del método científico. Se pretendió que los proyectos financiados fueran el enlace inicial con el desarrollo de una línea de trabajo sólida, que pueda participar a continuación en otros concursos como Fondecyt, Fondef, Fonis, etc. Las bases del concurso fueron publicadas en la página web de la sociedad ${ }^{1} \mathrm{y}$ en la Revista Chilena de Pediatría ${ }^{2}$. Dentro de los requisitos para participar, se destacó el hecho de que el investigador responsable y el coinvestigador debían ser miembros activos de SOCHIPE, permitiendo al primer autor participar en al menos un proyecto más como segundo autor o colaborador. También se permitió que entre los autores del proyecto pudiera incluirse a becados, alumnos o técnicos, para permitir la formación de recursos humanos jóvenes.

Dentro de los objetivos de promover la investigación clínica, está el incentivar la publicación científica en la Revista Chilena de Pediatría, principal publicación pediátrica a nivel nacional, sin desmedro de obtener publicaciones en revistas indexadas con índice de impacto tipo ISI.

Sin embargo, la mayor novedad en este concurso 2008 fue la creación de una Comisión de Investigación (CI) autónoma, de funcionamiento permanente, cuyas principales funciones corresponden a la evaluación y preselección de los proyectos postulados al concurso, la supervisión permanente del desarrollo de los proyectos financiados y la revisión de los informes técnicos de avance y finales de aquellos aprobados. Su responsabilidad principal es asegurar la calidad, transparencia y oportunidad de los procesos de evaluación y revisión de los proyectos. La CI se conformó por 5 miembros, designados por el Directorio de SOCHIPE, seleccionado personas que participan activamente en comités de investigaciones de otras instituciones científicas. Uno de los miembros es, 
adicionalmente, representante del Directorio de SOCHIPE.

Dentro de las directrices para el proceso de evaluación de proyectos, se consideró que la participación de los integrantes de la CI era a título personal, que la CI era responsable de velar por la calidad y rigurosidad de las evaluaciones externas, y que todos los proyectos que cumplieran con los requisitos establecidos en las bases fueran sometidos a arbitraje.

Como una manera de velar por la transparencia del concurso, se estableció que de haber algún proyecto participante en cuya autoría estuviese incluido algún integrante de la CI, éste sería examinado sin participación alguna del interesado, en ninguna de las etapas del proceso de evaluación. Cuando alguno de los integrantes de la CI tuvo conflicto de interés o relación de parentesco con cualquier postulante, se le invitó oportunamente a retirarse de la sala cada vez que se discutieron aspectos relacionados con dicho proyecto. Al igual que en los concursos de otras instituciones, como Fondecyt en Chile, Proyectos de la Comunidad Europea (FP-6, incodev y otros), NIH (Proyectos RO1 y otros) en Estados Unidos, la evaluación y asignación de árbitros se realizó en forma ciega al interesado. Adicionalmente, la CI no tiene la potestad de modificar las calificaciones asignadas por el árbitro externo. Sin embargo, cuando una calificación fue muy discordante entre los árbitros, se solicitó una tercera evaluación para definir el puntaje final. Como todo proceso de arbitraje científico, los integrantes de la CI tuvieron el deber de mantener en absoluta reserva toda la información que conocieron durante el ejercicio de su función, cualquiera sea la etapa del proceso de análisis y evaluación de los proyectos y sus respectivos informes.

Respecto al arbitraje, al término del proceso de evaluación todo proyecto contó con al menos 2 arbitrajes (un miembro de la CI y un evaluador externo), de acuerdo a su conocimiento del tema del proyecto y trayectoria académica y profesional. Algunos proyectos requirieron de 3 arbitrajes. Los evaluadores externos fueron investigadores activos, con productividad científica reciente en el área del proyecto de investigación. En lo posible, los evaluadores designados para cada proyecto fueron de distintas instituciones y de distintos grupos de trabajo. Quedan excluidos como evaluadores aquellos investigadores que temporalmente mantengan una situación pendiente con concursos anteriores, o a investigadores que tuviesen alguna relación de parentesco o que pertenecieran a la misma Unidad Académica de los postulantes.

El análisis de las evaluaciones, presupuesto y duración de los proyectos, fueron revisadas por la CI en reuniones programadas de acuerdo a calendario previo, con el propósito de conocer sus fortalezas y debilidades técnicas o teóricas, evaluar la calidad y pertinencia de las evaluaciones externas, y discrepar como grupo de las calificaciones externas, si ello era pertinente.

La CI envió al Directorio de SOCHIPE su evaluación final, la que fue discutida en reunión del Directorio con presencia del Presidente de la CI. Finalmente, los resultados del fallo fueron publicados en la página web de SOCHIPE$^{1}$ y comunicado a los ganadores en forma individual, por correo electrónico y convencional, con las sugerencias enviadas por los revisores. Los proyectos rechazados fueron comunicados a sus autores del mismo modo, dejando la posibilidad de que se solicitasen las evaluaciones de los revisores.

Este concurso de investigación año 2008, contó con $\$ 10000000$ (diez millones de pesos), con un máximo de $\$ 2000000$ (2 millones de pesos) por proyecto y se recepcionaron 17 Proyectos, 2 de los cuales quedaron excluidos por no completar los requisitos establecidos en las bases. Se financiaron las 5 primeras prioridades determinadas por su puntaje de acuerdo a la metodología descrita. Con posterioridad al inicio del concurso, la SOCHIPE logró obtener financiamiento adicional para otros 3 proyectos que, mereciendo financiamiento, habían quedado en las prioridades sexta, séptima y octava. Estos 3 proyectos fueron financiados con un $80 \%$ de lo solicitado. Posteriormente, uno de ellos fue retirado por el investigador principal, para perfeccionar su proyecto aprovechando las sugerencias arbitrales, para concursos venideros, lo que permitió financiar el $100 \%$ de los 2 proyectos restantes.

La CI cree que los proyectos beneficiados 
reflejan la diversidad de la temática de investigación pediátrica y reitera nuestras sinceras felicitaciones a los ganadores:

Screening de Desarrollo Psicomotor en la consulta pediátrica: Implementación de una prueba basada en el auto reporte de los padres, Dra. Luisa Schonhaut Berman.

Estudio de Hiperandrogenismo ovárico en pacientes puberales con Hiperplasia Suprarrenal congénita clásica por déficit de 21-Hidroxilasa, Dra. Francisca Ugarte Palacios.

Magnitud de la respuesta inmune humoral a la vacuna antihepatitis B en niños con cáncer recientemente diagnosticado: paso necesario para establecer recomendaciones nacionales de vacunación basadas en evidencia, Dr. Rodolfo Villena Martínez.

Interacciones Corazón-Pulmón en modelo pediátrico de Síndrome compartimental abdominal: influencia de la Compliance ToracoPulmonar y volumen tidal sobre la monitorización
Hemodinámica funcional y volumétrica de precarga, Dr. Franco Díaz Rubio.

Test de equilibrio peritoneal acortado en diálisis peritoneal pediátrica, Dr. Francisco Cano Sch.

Identificación del cromosoma 21 que predispone a su no disyunción y que daría cuenta de la alta prevalencia de Síndrome Down en Chile, Dr. Rodrigo Moreno Salinas.

Evaluación de la función de la glándula suprarrenal en pacientes con Síndrome bronquial obstructivo tratados desde 5 hasta 7 días con corticoides sistémicos, Dr. Alejandro Martínez Aguayo.

\section{Referencias}

1.- Pagina Web Sociedad Chilena de Pediatría: www. sochipe.cl.

2.- Concurso de Proyectos de Investigación 2008, Sociedad Chilena de Pediatría. Rev Chil Pediatr 2008; 79 (1): 107-8. 